\title{
End Point Force Control of a Flexible Timoshenko Arm
}

\author{
Minoru Sasaki', Kouki Nagaya1, Takahiro Endo ${ }^{2}$, Kojiro Matsushita1 ${ }^{1}$, Satoshi Ito ${ }^{1}$ \\ ${ }^{1}$ Department of Mechanical Engineering, Gifu University, Gifu, Japan \\ ${ }^{2}$ Department of Mechanical Engineering and Science, Graduate School of Engineering, Kyoto University, Kyoto, \\ Japan \\ Email: sasaki@gifu-u.ac.jp, u3128026@edu.gifu-u.ac.jp, endo@me.kyoto-u.ac.jp, \\ kojirom@gifu-u.ac.jp,satoshi@gifu-u.ac.jp
}

Received September 2015

\section{Abstract}

This paper discusses a force control problem for a flexible Timoshenko arm. The effect of shear deformation and the effect of rotary inertia are considered in Timoshenko beam theory. Most of the research about force control of the flexible arm is based on Euler Bernoulli beam theory. There are a few researches about force control of the flexible arm using Timoshenko beam theory. The aim of the force control is to control the contact force at the contact point. To solve this problem, we propose a simple controller using Timoshenko beam theory. Finally, we describe simulation results using a numerical inversion of Laplace transform carried out to investigate the validity of the proposed controller for the force control problem. The results of the time response show the transverse displacement, the angle of deflection, the slider position, the rotational angle and the contact force toward the desired their values.

\section{Keywords}

Flexible Arm, Timoshenko Beam Theory, Force Control, Distributed Parameter Systems Control, Laplace Transform

\section{Introduction}

In recent years, there has been a great deal of interest in the modeling and control of flexible arms [1]-[11]. This interest has been motivated by the prospect of fast, light, robot whose links, due to material characteristics, will bend under heavy loads. As a first step towards designing controllers for such robots, researchers have begun studying controllers for simple flexible links. These links, in most cases modeled as Euler-Bernoulli beams because of the small deflections involved, are often analyzed through an eigen-function series expansion of the solution to beam equation. However, under author's knowledge, there has not yet been a study of force control of a flexible Timoshenko arm based on the infinite dimensional model. The effect of shear deformation and the effect of rotary inertia are considered in Timoshenko beam theory and thus the Timoshenko beam theory is modified for a non-slender beam and high-frequency response. This means that the Timoshenko beam theory 
has a wider application area than the Euler-Bernoulli beam theory. So we discuss the force control problem for the flexible Timoshenko arm.

\section{Equations of Motion and Boundary Conditions}

Figure 1 shows a constrained one-link flexible Timoshenko arm. One-end of the arm is clamped to control actuators consisting of the rotational motor and the translational slider, and the other end has a concentrated tip mass $m$. The tip mass makes contact with the surface of an object. The flexible arm translates and rotates in the horizontal plane (the $X Y$ plane in Figure 1) by control actuators; it is not affected by the acceleration of gravity. The flexible arm, with length $l$, mass per unit length $\rho$, mass moment of inertia $I_{\rho}$, cross sectional area $A$, area moment of inertia $I$, Young's modulus $E$, shear modulus $G$, and shear coefficient $\kappa$, satisfies the Timoshenko beam hypothesis.

In Figure 1, $X Y$ is an absolute coordinate system and $x y$ is a local coordinate system, whose origin is fixed at the rotor of the rotational motor. In addition, $x y$ translates with the slider and rotates with the rotor of the motor. Let $J, \tau_{m}(t), \theta(t), M, F_{s}(t)$, and $s(t)$ be the inertia moment of the motor, the torque generated by the motor at time $t$, the rotational angle of the motor, the mass of the slider, the force generated by the slider, and the translational position of the slider, respectively. Further, let $w(x, t)$ and $\phi(x, t)$ be the transverse displacement of the flexible arm at time $t$ and spatial point $x$, and the rotation of the cross section due to bending deformation, respectively.

Since the tip mass makes contact with the surface of the object, we obtain the following geometric constraint:

$$
\Phi \equiv l \theta(t)+w(l, t)+s(t)=0 .
$$

This constraint means that the $Y$-axis position of the tip mass is constrained on the surface of the object. The kinetic energy $E_{K}$ and the potential energy $E_{P}$ of the overall system are given by the following:

$$
\begin{gathered}
E_{K}=\frac{\rho}{2} \int_{0}^{l}[x \dot{\theta}(t)+\dot{w}(x, t)+\dot{s}(t)]^{2} \mathrm{~d} x+\frac{J}{2} \dot{\theta}^{2}(t)+\frac{M}{2} \dot{s}^{2}(t) \\
+\frac{I \rho}{2} \int_{0}^{l}[\dot{\theta}(t)+\dot{\phi}(x, t)]^{2} \mathrm{~d} x+\frac{m}{2}[l \dot{\theta}(t)+\dot{w}(l, t)+\dot{s}(t)]^{2}, \\
E_{P}=\frac{E I}{2} \int_{0}^{l}\left[\phi^{\prime}(x, t)\right]^{2} \mathrm{~d} x+\frac{K}{2} \int_{0}^{l}\left[\phi(x, t)-w^{\prime}(x, t)\right]^{2} \mathrm{~d} x,
\end{gathered}
$$

where $K=\kappa G A$, a dot denotes the time derivative, and a prime denotes the partial derivative with respect to $x$. Here the virtual work is given by $\delta W=\tau(t) \delta \theta(t)+F(t) \delta s(t)$.

Under the above preparation, we can obtain the following equations of motion by applying Hamilton's principle and Lagrange's multiplier, and using the procedure described in [13]:

$$
\begin{gathered}
\rho[\ddot{w}(x, t)+x \ddot{\theta}(t)+\ddot{s}(t)]+K\left[\phi^{\prime}(x, t)-w^{\prime \prime}(x, t)\right]=0, \\
I_{\rho}[\ddot{\phi}(x, t)+\ddot{\theta}(t)]+K\left[\phi(x, t)-w^{\prime}(x, t)\right]-E I \phi^{\prime \prime}(x, t)=0, \\
w(0, t)=\phi(0, t)=\phi^{\prime}(l, t)=l \theta(t)+w(l, t)+s(t)=0, \\
J \ddot{\theta}(t)=\tau_{m}(t)+E I \phi^{\prime}(0, t) \equiv \tau(t),
\end{gathered}
$$

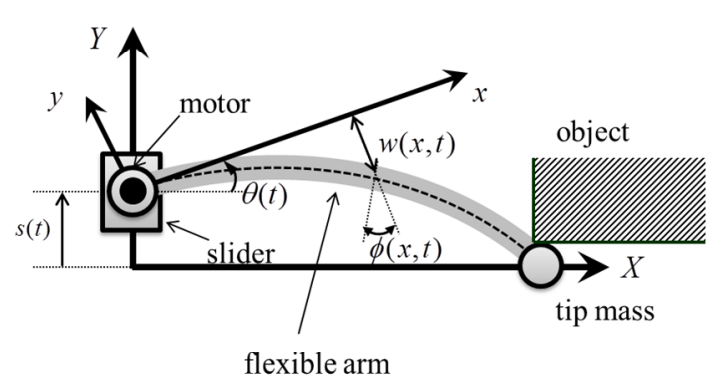

Figure 1. Flexible Timoshenko arm making contact with an object. 


$$
M \ddot{s}(t)=F_{s}(t)+K w^{\prime}(0, t) \equiv F(t),
$$

with the algebraic relation

$$
\lambda(t)=K\left[w^{\prime}(l, t)-\phi(l, t)\right],
$$

where $\lambda(t)$ is Lagrange's multiplier and is equivalent to the contact force, i.e., the shear force at the tip of the flexible arm, which arises in the direction along the normal vector of the constraint surface.

The aim of this paper is to control the contact force at the tip of the flexible arm. In other words, the control objective is to construct a boundary controller satisfying

$$
\left\{\begin{array}{l}
\lambda(t) \rightarrow \lambda_{d}, \quad \dot{w}(x, t) \rightarrow 0, \quad \dot{\phi}(x, t) \rightarrow 0, \\
\dot{\theta}(t) \rightarrow 0, \quad \dot{s}(t) \rightarrow 0,
\end{array}\right.
$$

where $\lambda_{d}$ is the constant desired contact force. At the desired equilibrium point

$\left(\lambda(t)=\lambda_{d}, \dot{w}(x, t)=\dot{\phi}(x, t)=\dot{\theta}(t)=\dot{s}(t)=0\right), w(x, t)$ and $\phi(x, t)$ become the function of the variable $x$, and $\theta(t)$ and $s(t)$ become constant. Thus, we describe them as $w_{d}(x), \phi_{d}(x), \theta_{d}$, and $s_{d}$, respectively. By substituting these into (1)-(6), we obtain:

$$
\left\{\begin{array}{l}
w_{d}(x)=\lambda_{d} x\left(\frac{1}{K}+\frac{l x}{2 E I}-\frac{x^{2}}{6 E I}\right), \phi_{d}(x)=\frac{\lambda_{d} x}{E I}\left(l-\frac{x}{2}\right), \\
l \theta_{d}+s_{d}=-\lambda_{d} l\left(\frac{1}{K}+\frac{l^{2}}{3 E I}\right)
\end{array}\right.
$$

In these relations, $w_{d}(x), \phi_{d}(x), \theta_{d}$, and $s_{d}$ mean a static transverse displacement, a static rotation of the cross section of the flexible arm, a static angle of the motor, and a static position of the slider in the case where the contact force is converged to the desired value, respectively. Furthermore, $\theta_{d}$ and $s_{d}$ are coupled through $\lambda_{d}$, and thus we cannot set the desired angle $\theta_{d}$ and the desired position $s_{d}$ independently.

Based on these results, we set the control objective as follows: to construct a controller accomplishing:

$$
\left\{\begin{array}{l}
w(x, t) \rightarrow w_{d}(x), \dot{w}(x, t) \rightarrow 0, \\
\phi(x, t) \rightarrow \phi_{d}(x), \dot{\phi}(x, t) \rightarrow 0, \\
\theta(t) \rightarrow \theta_{d}, \dot{\theta}(t) \rightarrow 0, s(t) \rightarrow s_{d}, \dot{s}(t) \rightarrow 0 .
\end{array}\right.
$$

For this purpose, we propose the following controller:

$$
\begin{aligned}
& F(t)=\tilde{k}_{1} K\left[w^{\prime}(0, t)-w_{d}^{\prime}(0)\right]+\tilde{k}_{2} K \dot{w}^{\prime}(0, t)-\tilde{k}_{3}\left[s(t)-s_{d}\right]-\tilde{k}_{4} \dot{s}(t), \\
& \tau(t)=\tilde{k}_{5} E I\left[\dot{\phi}(0, t)-\dot{\phi}_{d}(0)\right]+\tilde{k}_{6} E I \dot{\phi}^{\prime}(0, t)-\tilde{k}_{7}\left[\theta(t)-\theta_{d}\right]-\tilde{k}_{8} \dot{\theta}(t),
\end{aligned}
$$

where feedback gain $\tilde{k}_{i}, i=1, \ldots, 8$, is a positive constant. In these controllers, $(10)$ is the controller for the slider and (11) is the controller for the motor. In (10), the first and second terms are for the control: $w(x, t) \rightarrow w_{d}(x)$ and $\dot{w}(x, t) \rightarrow 0$, and the third and the forth terms are for the position control: $s(t) \rightarrow s_{d}$ and $\dot{s}(t) \rightarrow 0$. On the other hand, in (11), the first and second terms are for the control of the rotation of the cross section: $\phi(x, t) \rightarrow \phi_{d}(x)$ and $\dot{\phi}(x, t) \rightarrow 0$, and the third and the forth terms are for $\theta(t) \rightarrow \theta_{d}$ and $\dot{\theta}(t) \rightarrow 0$. Here, note that if we use the strain gauge, rotary encoder, and speed reference type servo amplifier of the motor and the slider, we can easily implement the controller.

\section{Laplace Transform of the Equation of Motion}

Taking the Laplace transform of (1)-(11), we can get

$$
\begin{gathered}
s^{2} \rho w(x, s)+K\left(\phi^{\prime}(x, s)\right)-K\left(w^{\prime \prime}(x, s)\right)+s^{2} \rho(x \theta(s)+S(s))=0 \\
\left(s^{2} I_{\rho}+K\right) \phi(x, s)-K w^{\prime}(x, s)-E I \phi^{\prime \prime}(x, s)+s^{2} I_{\rho} \theta(s)=0 \\
w(0, s)=0
\end{gathered}
$$




$$
\begin{gathered}
\phi(0, s)=0 \\
\phi^{\prime}(l, s)=0 \\
l \theta(s)+w(l, s)+S(s)=0 \\
s^{2} J \theta(s)=\tau_{m}(s)+E I \phi^{\prime}(0, s) \equiv \tau(s) \\
s^{2} M S(s)=F_{s}(s)+K w^{\prime}(0, s) \equiv F(s) \\
F(s)=\left(\tilde{k}_{1}+s \tilde{k}_{2}\right) K w^{\prime}(0, s)-\left(\tilde{k}_{3}+s \tilde{k}_{4}\right) S(s)-\frac{1}{s}\left(\tilde{k}_{1} K w_{d}^{\prime}(0)+\tilde{k}_{3} S_{d}\right) \\
\tau(s)=\left(\tilde{k}_{5}+s \tilde{k}_{6}\right) E I \phi^{\prime}(0, s)-\left(\tilde{k}_{7}+s \tilde{k}_{8}\right) \theta(s)-\frac{1}{s}\left(\tilde{k}_{5} E I \phi_{d}^{\prime}(0)-\tilde{k}_{7} \theta_{d}\right)
\end{gathered}
$$

Finally we get $w(0, s)$ and $\phi(0, s)$ as follows:

$$
\begin{aligned}
& w(0, s)=\frac{1}{4} \frac{1}{K^{2} s\left(s k_{4}+s^{2}+k_{3}\right)\left(k_{7}+s k_{8}+s^{2}\right) E \rho I}(\sqrt{2}(-F 2(s)+F 1(s)) \\
& *\left(-\left(s k_{4}+s^{2}+k_{3}\right)\left(k_{7}+s k_{8}+s^{2}\right)\right. \\
& *\left(-\sqrt{s^{2} \rho^{2} E^{2} I^{2}-4 K E \rho\left(\frac{1}{2} s^{2} I_{\rho}+K\right) I+K^{2} s^{2} I_{\rho}{ }^{2}}+s\left(E I_{\rho}-I_{\rho} K\right)\right. \\
& +\left(k_{8}+k_{4}\right)\left(E I_{\rho}-I_{\rho} K\right) s^{3}+\left(E \rho\left(-2 K^{2} k_{2} k_{6}+k_{3}+k_{4} k_{8}+k_{7}\right) I\right. \\
& -I_{\rho} K\left(k_{3} J+k_{4} k_{8}+M k_{7}\right) s^{2}-2 E\left(\left(k_{1} k_{6}+k_{2} k_{5}\right) K^{2}-\frac{1}{2} k_{3} k_{8}-\frac{1}{2} k_{4} k_{7}\right) \rho I \\
& \left.\left.\left.-K I_{\rho}\left(k_{3} k_{8}+k_{4} k_{7}\right)\right) s-2 E\left(-\frac{1}{2} k_{3} k_{7}+K^{2} k_{1} k_{5}\right) \rho I-K I_{\rho} k_{3} k_{7}\right)\right) \\
& * \sqrt{K E I\left(s \rho E I+K s I_{\rho}-\sqrt{s^{2} \rho^{2} E^{2} I^{2}-4 K E \rho\left(\frac{1}{2} s^{2} I_{\rho}+K\right) I+K^{2} s^{2} I_{\rho}^{2}}\right) s} \\
& +\sqrt{2}\left(s k_{4}+s^{2}+k_{3}\right)\left(k_{7}+s k_{8}+s^{2}\right) \\
& * \sqrt{s^{2} \rho^{2} E^{2} I^{2}-4 K E \rho\left(\frac{1}{2} s^{2} I_{\rho}+K\right) I+K^{2} s^{2} I_{\rho}^{2}}+s\left(E I_{\rho}-I_{\rho} K\right) \\
& +\left(k_{8}+k_{4}\right)\left(E I_{\rho}-I_{\rho} K\right) s^{3}+\left(E \rho\left(-2 K^{2} k_{2} k_{6}+k_{3}+k_{4} k_{8}+k_{7}\right) I\right. \\
& -I_{\rho} K\left(k_{3}+k_{4} k_{8}+k_{7}\right) s^{2}-2 E\left(\left(k_{1} k_{6}+k_{2} k_{5}\right) K^{2}-\frac{1}{2} k_{3} k_{8}-\frac{1}{2} k_{4} k_{7}\right) \rho I \\
& \left.\left.\left.-K I_{\rho}\left(k_{3} k_{8}+k_{4} k_{7}\right)\right) s-2 E\left(-\frac{1}{2} k_{3} k_{7}+K^{2} k_{1} k_{5}\right) \rho I-K I_{\rho} k_{3} k_{7}\right)\right) \\
& +2 E I K\left(-s^{2}(-F 3(s)+F 2(s)+F 1(s)-F 4(s))\left(k_{1}+s k_{2}\right)\left(k_{7}+s k_{8}+s^{2}\right) *\right. \\
& \sqrt{s^{2} \rho^{2} E^{2} I^{2}-4 K E \rho\left(\frac{1}{2} s^{2} I_{\rho}+K\right) I+K^{2} s^{2} I_{\rho}^{2}} \\
& +\left(s\left(k_{1}+s k_{2}\right)\left(k_{7}+s k_{8}+s^{2}\right) *\left(\left(E I_{\rho}-I_{\rho} K\right) s^{2}-2 K^{2}\right)\right) \\
& *(F 1(s)+F 2(s)+F 3(s)+F 4(s))+2\left(\left(k_{1} \lambda_{d}+k_{3} S_{d}\right) s^{2}+\left(-k_{2}\left(k_{5} \lambda_{d} l-k_{7} \theta_{d}\right) K\right.\right. \\
& \left.\left.\left.+k_{8}\left(k_{1} \lambda_{d}+k_{3} S_{d}\right)\right) s-k_{1}\left(-k_{2}\left(k_{5} \lambda_{d} l-k_{7} \theta_{d}\right) K+k_{7}\left(k_{1} \lambda_{d}+k_{3} S_{d}\right)\right) K\right) \rho\right)=0,
\end{aligned}
$$




$$
\begin{aligned}
& \phi(0, s)=-\frac{1}{2} \frac{1}{K s\left(k_{7}+s k_{8}+s^{2}\right)}\left(-s \sqrt{2}(-F 2(s)+F 1(s))\left(k_{5}+s k_{6}\right)\right. \\
& * \sqrt{K E I\left(s \rho E I+K s I_{\rho}-\sqrt{s^{2} \rho^{2} E^{2} I^{2}-4 K E \rho\left(\frac{1}{2} s^{2} I_{\rho}+K\right) I+K^{2} s^{2} I_{\rho}^{2}}\right)} s-s \sqrt{2}(-F 4(s)+F 3(s)) \\
& \left(k_{5}+s k_{6}\right) * \sqrt{K E I\left(s \rho E I+K s I_{\rho}+\sqrt{s^{2} \rho^{2} E^{2} I^{2}-4 K E \rho\left(\frac{1}{2} s^{2} I_{\rho}+K\right) I+K^{2} s^{2} I_{\rho}^{2}}\right)} \\
& \left.+2 K\left(k_{5} \lambda_{d} l-k_{7} \theta_{d}\right)\right)+F 1(s)+F 2(s)+F 3(s)+F 4(s)=0,
\end{aligned}
$$

where the constants $F 1(s), F 2(s), F 3(s)$ and $F 4(s)$ can be determined using the boundary conditions.

\section{Numerical Simulation Results}

Numerical inversion of Laplace transform is used to obtain the results in the time domain. The computation of the inverse Laplace transform is based on the paper of T. Hosono [12]. In the computer simulation study, we consider a typical arm whose parameters are given in the Table $\mathbf{1}$. To investigate the validity of the proposed controller, we considered the step responses of the desired contact force, $\lambda_{d}=-100 \mathrm{~N}$, and the desired position of the slider, $s_{d}=0.1 \mathrm{~m}$. Here, note that $\lambda_{d}=-100$ means that the flexible arm pushed the surface of the environment by the force of 100 N. Figures 2-6 show the time response of the transverse displacement, the angle of deflection, the slider position, the rotational angle and the contact force with adequate feedback gains $k_{1}=k_{5}=1, k_{2}=k_{6}=0.2, k_{3}=k_{7}=16, k_{4}=k_{8}=8$ using try and error method. The desired value of $w_{d}$, $\phi_{d}, \theta_{d}$ are

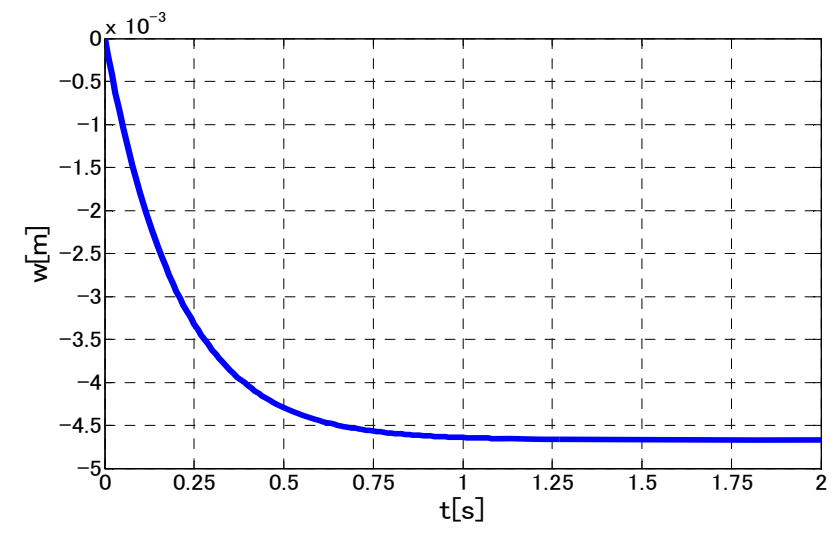

Figure 2. Time response of transverse displacement of the arm.

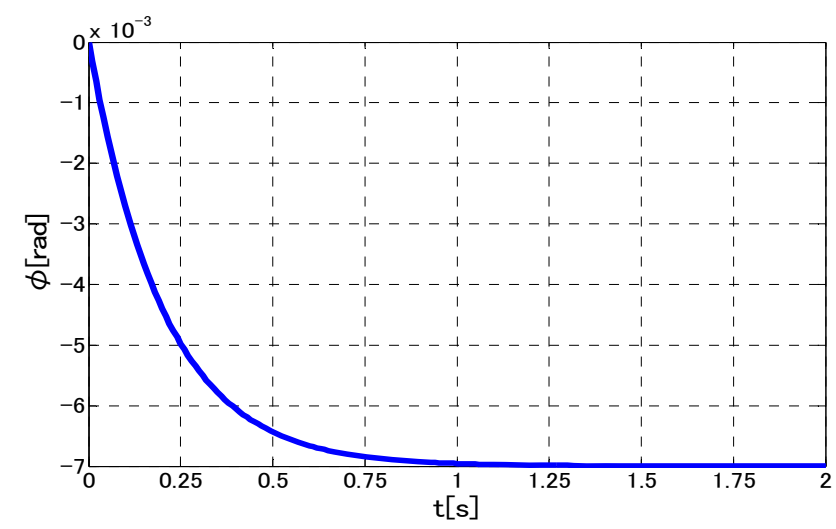

Figure 3. Time response of angle of deflection of the arm. 


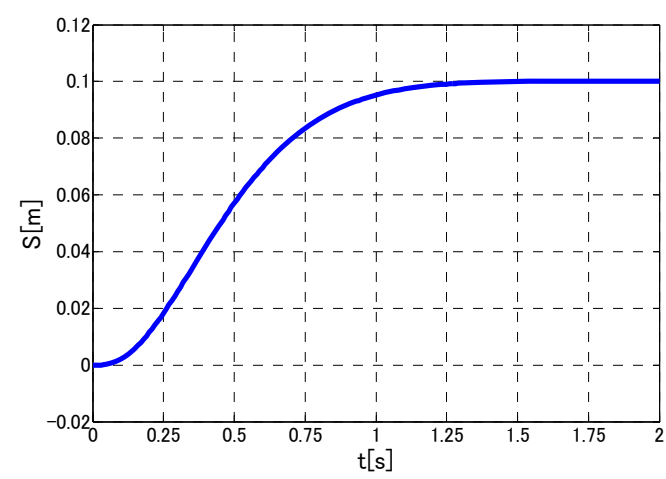

Figure 4. Time response of the slider position.

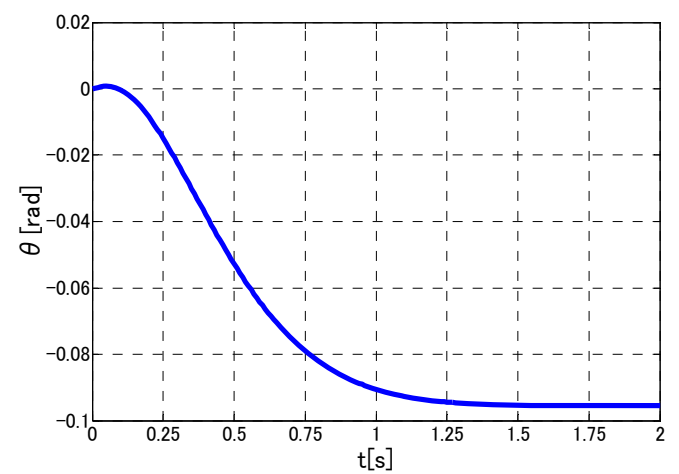

Figure 5. Time response of rotational angle.

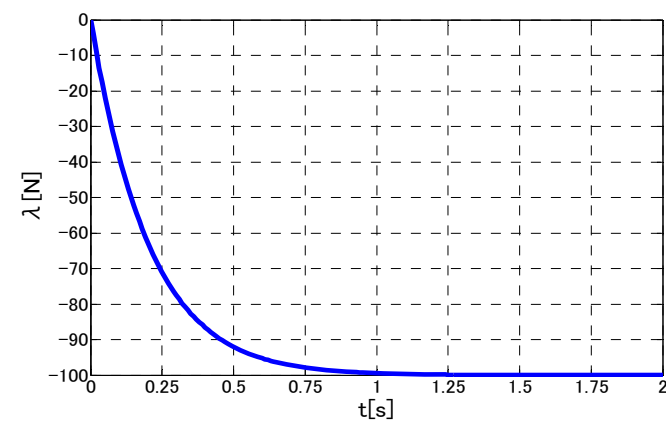

Figure 6. Time response of the contact force.

Table 1. System parameters.

\begin{tabular}{ccc}
\hline Length & $l$ & $1.0[\mathrm{~m}]$ \\
Mass per unit length & $\rho$ & $2.73[\mathrm{~kg} / \mathrm{m}]$ \\
Mass moment of inertia & $I_{\rho}$ & $2.79 \mathrm{e}(-4)[\mathrm{kgm}]$ \\
Cross sectional area & $A$ & $3.5 \mathrm{e}(-4)\left[\mathrm{m}^{4}\right]$ \\
Area moment of inertia & $I$ & $3.57 \mathrm{e}(-8)\left[\mathrm{m}^{4}\right]$ \\
Young's modulus & $E$ & $2.00 \mathrm{e} 11[\mathrm{~Pa}]$ \\
Shear modulus & $G$ & $7.69 \mathrm{e} 10[\mathrm{~Pa}]$ \\
Shear coefficient & $\kappa$ & $5 / 6$ \\
Desired contact force & $\lambda_{d}$ & $-100[\mathrm{~N}]$ \\
Desired translational position of the slider & $S_{d}$ & $0.1[\mathrm{~m}]$ \\
\hline
\end{tabular}




$$
\left\{\begin{array}{c}
\phi_{d}(x)=-7.00 \times 10^{-3}[\mathrm{rad}] \\
w_{d}(x)=-4.67 \times 10^{-3}[\mathrm{~m}] \\
\theta_{d}=-0.0953[\mathrm{rad}]
\end{array}\right.
$$

It can be seen that the transverse displacement, the angle of deflection, the slider position, the rotational angle and the contact force toward the desired their values. With the adequate feedback gains there are no residual vibrations and no over shoot.

\section{Conclusion}

A contact-force control problem with regards to a constrained one-link flexible Timoshenko arm was described. The equations of motion and the boundary conditions of the overall system were derived. To solve the contact force control problem of such a system, we have proposed a simple controller, which is easy to implement. Several numerical simulations using a numerical inversion of Laplace transform were carried out. The simulation results showed the validity of the proposed controller for the contact-force control problem with no residual vibrations and no overshoot.

\section{References}

[1] Yuan, K. and Hu, C.-M. (1996) Nonlinear Modeling and Partial Linearizing Control of a Slewing Timoshenko-Beam. Trans. ASME Journal of Dynamic Systems, Measurement, and Control, 118, 75-83. http://dx.doi.org/10.1115/1.2801154

[2] Tadi, M. (1997) Comparison of Two Finite-Element Schemes for Feedback Control of a Timoshenko Beam. Proceedings of the ASME Dynamic Systems and Control Division, 61, 587-596.

[3] White, M.H.D. and Heppler, G.R. (1996) Vibration of a Rotating Timoshenko Beam. Trans. ASME Journal of Vibration and Acoustic, 118, 606-613. http://dx.doi.org/10.1115/1.2888341

[4] Sasaki, M., Ueda, T., Inoue, Y. and Book, W.J. (2012) Passivity-Based Control of Rotational and Translational Timoshenko Arms. Advances in Acoustics and Vibration, 2012, Article ID: 174816. http://dx.doi.org/10.1155/2012/174816

[5] Morgül, Ö. (1992) Dynamic Boundary Control of the Timoshenko Beam. Automatica, 28, 1255-1260. http://dx.doi.org/10.1016/0005-1098(92)90070-V

[6] Oguamanam, D.C.D. and Heppler, G.R. (1996) The Effect of Rotating Speed on the Flexural Vibration of a Timoshenko Beam. Proc. of the 1996 IEEE International Conference on Robotics and Automation, 2438-2443. http://dx.doi.org/10.1109/ROBOT.1996.506529

[7] Zhang, F., Dawson, D.M., de Queiroz, M.S. and Vedagarbha, P. (1997) Boundary Control of the Timoshenko Beam with Free-End Mass/Inertial Dynamics. Proc. of the 36th IEEE Conference on Decision \& Control, 245-250. http://dx.doi.org/10.1109/CDC.1997.650623

[8] Taylor, S.W. and Yau, S.C.B. (2003) Boundary Control of a Rotating Timoshenko Beam. ANZIAM J., 44, E143-E184.

[9] Rastgoftar, H., Mahmoodi, M., Eghtesad, M. and Kazemi, M. (2008) Stability Analysis of a Flexible Two-Link Timoshenko Manipulator Using Boundary Control Method. Proc. of ASME 2008 International Mechanical Engineering Congress and Exposition, 409-415.

[10] Grobbelaar-Van Dalsen, M. (2010) Uniform Stability for the Timoshenko Beam with Tip Load. J. Math. Anal. Appl., 361, 392-400. http://dx.doi.org/10.1016/j.jmaa.2009.06.059

[11] Han, Z.J. and Xu, G.Q. (2011) Dynamical Behavior of a Hybrid System of Nonhomogeneous Timoshenko Beam with Partial Non-Collocated Inputs. J. Dyn. Control Syst., 17, 77-121. http://dx.doi.org/10.1007/s10883-011-9111-6

[12] Hosono, T. (1981) Numerical Inversion of Laplace Transform and Some Application to Wave Optics. Radio Science, 16, 1015. http://dx.doi.org/10.1029/RS016i006p01015

[13] Endo, T., Matsuno, F. and Kawasaki, H. (2014) Force Control and Exponential Stabilisation of One-Link Flexible Arm. Int. J. Control, 87, 1794-1807. http://dx.doi.org/10.1080/00207179.2014.889854 